Research Article

\title{
Fuzzy Evaluation of Pharmacokinetic Models
}

\author{
Carlos Sepúlveda $\left(\mathbb{D},{ }^{1}\right.$ Oscar Montiel $\left(\mathbb{D},{ }^{1}\right.$ José M. Cornejo Bravo $\mathbb{D}^{2}{ }^{2}$ \\ and Roberto Sepúlveda ${ }^{1}{ }^{1}$ \\ ${ }^{1}$ Instituto Politécnico Nacional, Centro de Investigación y Desarrollo de Tecnología Digital (CITEDI-IPN), \\ Av. Instituto Politécnico Nacional, No. 1310, Col. Nueva Tijuana, 22435 Tijuana, BC, Mexico \\ ${ }^{2}$ Facultad de Ciencias Químicas e Ingeniería, Universidad Autónoma de Baja California, Calzada Universidad 14418, \\ Parque Industrial Internacional Tijuana, 22390 Tijuana, BC, Mexico
}

Correspondence should be addressed to Oscar Montiel; oross@ipn.mx

Received 4 July 2018; Revised 8 September 2018; Accepted 19 September 2018; Published 1 November 2018

Guest Editor: Fevrier Valdez

Copyright ( 2018 Carlos Sepúlveda et al. This is an open access article distributed under the Creative Commons Attribution License, which permits unrestricted use, distribution, and reproduction in any medium, provided the original work is properly cited.

\begin{abstract}
Population pharmacokinetic (PopPK) models allow researchers to predict and analyze drug behavior in a population of individuals and to quantify the different sources of variability among these individuals. In the development of PopPK models, the most frequently used method is the nonlinear mixed effect model (NLME). However, once the PopPK model has been developed, it is necessary to determine if the selected model is the best one of the developed models during the population pharmacokinetic study, and this sometimes becomes a multiple criteria decision making (MCDM) problem, and frequently, researchers use statistical evaluation criteria to choose the final PopPK model. The used evaluation criteria mentioned above entail big problems since the selection of the best model becomes susceptible to the human error mainly by misinterpretation of the results. To solve the previous problems, we introduce the development of a software robot that can automate the task of selecting the best PopPK model considering the knowledge of human expertise. The software robot is a fuzzy expert system that provides a method to systematically perform evaluations on a set of candidate PopPK models of commonly used statistical criteria. The presented results strengthen our hypothesis that the software robot can be successfully used to evaluate PopPK models ensuring the selection of the best PopPK model.
\end{abstract}

\section{Introduction}

Pharmacokinetics (PK) is a subdivision of pharmacology in which mathematical models are developed to study the processes of absorption, distribution, and elimination of a drug once a given dose has been administered to a given individual [1]. These mathematical models are derived from representing the body as a system of compartments [2] (Figure 1), where the transfer rates of absorption, distribution, redistribution, and elimination between these compartments can be used to determine the parameters of the PK model such as clearance $(\mathrm{Cl})$ and volume of distribution $(V)$ that allow us to predict the drug concentration in plasma $\left(C_{\mathrm{p}}\right)$ in a given time [3].

Let us consider the one compartment PK model of Figure 1, where the rate of elimination of the drug presented in the body decreases in proportion to the $C_{p}$, that is, with a first-order elimination process $k_{\mathrm{e}}$ and the drug administered as a single intravenous bolus dose $(D)$; the kinetic of $C_{p}$ in the compartment-the body-at time $t>0$ is depicted by the following deterministic differential equation:

$$
\begin{aligned}
\frac{d C_{\mathrm{p}_{t}}}{d t} & =-k_{\mathrm{e}} C_{\mathrm{p}_{t}}, \\
C_{\mathrm{p}_{0}} & =\frac{D}{V} .
\end{aligned}
$$

In this case, the term $k_{\mathrm{e}}$ is given by

$$
k_{\mathrm{e}}=\frac{\mathrm{Cl}}{V},
$$

where $V$ is the distribution volume of the drug within the body and $\mathrm{Cl}$ measures the ability of the liver and the kidney, mainly to extract a drug from the body. In this context, $\mathrm{Cl}$ and $V$ are fixed parameters that describe the drug concentration of a given dose $D$ over time $t$. Equation (1) has the following explicit solution: 


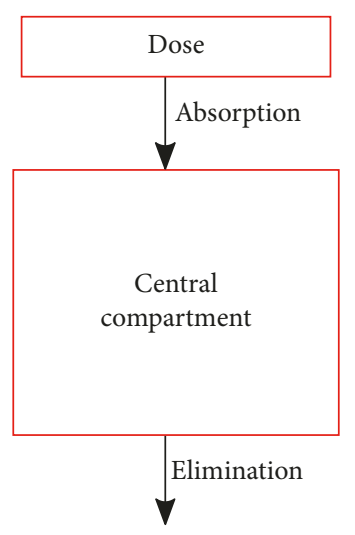

(a)

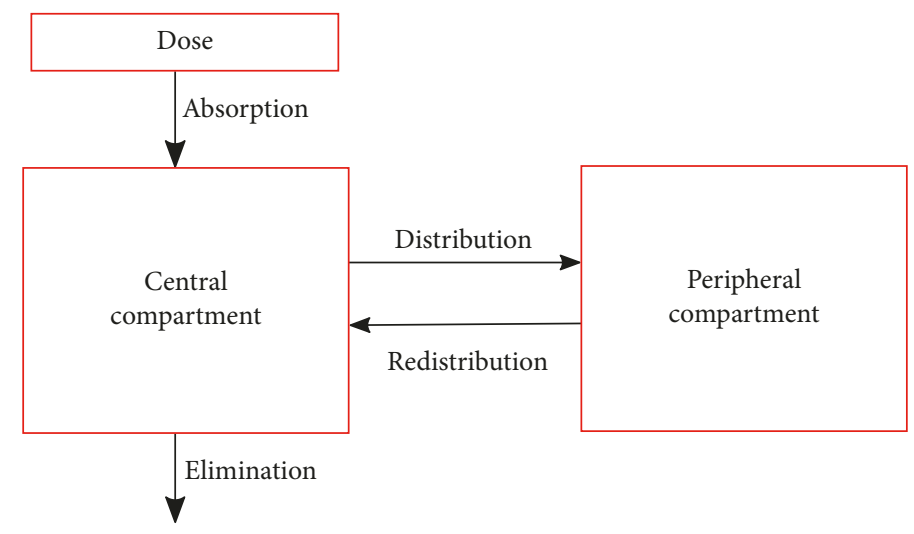

(b)

FIGURE 1: Example of the body viewed as (a) one-compartment model or (b) two-compartment model. The central compartment represents the plasma and tissues, and the peripheral compartment represents those tissues that take up the drug at a slower rate.

$$
C_{\mathrm{p}_{t}}=\frac{D}{V} e^{-(\mathrm{Cl} / V) \cdot t}
$$

The model (3) states a relationship between the independent variable $t$ and the dependent variable $C_{p}[1,4]$.

PopPK models are designed to analyze drug behavior in a group of individuals; hence, it is possible to generalize the model for similar individuals that were not subject of the study. NLME modeling is the main current approach for PopPK model development; it allows estimation of parameters in the presence of different levels of variability, and it is commonly used when it is not possible to obtain the complete information on repeated measurements of individuals [2]. Most of the time, the process to find the final PopPK model that better represents the data behavior requires the development of several PopPK models. It is common to use interchangeable software programs like $\mathrm{R}$ or SAS to apply various evaluation criteria to select which PopPK model is "better" among all the possible PopPK models [5-7]. These evaluation criteria range from graphical analysis to statistical methods [8]. The graphical analysis is limited to expert modelers, and besides that, it can be time-consuming and is subject to human error or misinterpretation.

The statistical methods such as the estimated error variance (MSE), the objective function value (OFV), the Akaike's information criterion (AIC), or the Bayesian information criterion (BIC), also known as Schwarz criterion (CS) offer quantitative terms (values) which are much easier to interpret and for consequence more easily to exclude or accept. However, the respective values may be contrasted between PopPK models when more than one statistical criterion is used complicating in this way ensuring the selection of the best PopPK model, thus becoming a multiple criteria decision making (MCDM) problem [9].

Fuzzy expert systems is an area where artificial intelligence and fuzzy logic meet to make robots backing or even replace human experts that want to do automated tasks [10]. The fuzzy logic has been recognized as an essential problem-solving technique when human evaluations are required for interpretation of medical findings [11], as well as in problems where is necessary for managing data with uncertainty such as [12], where a type-2 fuzzy logic methodology is used to help a neural network to handle complex time series data.

We contribute with a new software robot to facilitate the selection of the best pharmacokinetic model by an automated process when the comparison of the implemented evaluation criteria values among the models cannot be categorically determined, addressing the problem as a decision problem. We present results using a software robot to select the best PopPK model of tobramycin by an automated process.

The organization of this work is as follows: in Section 2, an overview of nonlinear mixed-effects modeling is provided. In Section 3, the implementation of the software robot is explained. In Section 4, the parameter estimation results and the outcomes of the software robot are presented. In Section 5, an analysis of the results is exposed. Finally, in Section 6, the conclusions are given.

\section{Nonlinear Mixed-Effects Model Framework}

In mixed-effects models, the data set is longitudinal, i.e., it is composed of repeated samples of the same individuals $[13,14]$. This is the case of the PopPK experimental data; it consists of a vector $y_{i j}$ of samples of the $j-$ th measurement (where, $j=1, \ldots, n$ ) of $C_{p}$ in the $i$-th individual (where, $i=1, \ldots, n$ ). Therefore, we can obtain the sample $y_{i j}$ for the individual $i$ at the time $t_{i j}$ by using the model:

$$
y_{i j}=f\left(x_{i j}, \phi_{i}\right)+\varepsilon_{i j} ; \quad \varepsilon_{i j} \sim\left(0, \sigma^{2}\right),
$$

where $f(\cdot)$ is a nonlinear function as Equation (3) relating a vector of known values $x_{i j}$ (e.g., dose and time) to the unknown parameter vector $\phi_{i}$ (e.g., $\left.\left(\mathrm{Cl}_{i}, V_{i}\right)^{\mathrm{T}}\right)$ and the variable $\varepsilon_{i j}$ in the second term is the residual error that is assumed to be normally distributed with mean zero and variance $\sigma^{2}$. It is sensible to expect that each individual has a different parameter vector $\phi_{i}$, and this is described in a second stage by the covariate model: 


$$
\phi_{i}=g\left(z_{i} ; \theta\right)+\eta_{i} ; \quad \eta_{i} \sim\left(0, \omega^{2}\right) .
$$

Equation (5) describes the variation among different individuals accounted through the individual-specific parameters $\phi_{i}$.

The function $g(\cdot)$ relates the specific covariates $z_{i}$ of the individuals to the population parameters $\theta$. $\eta_{i}$ is a $(q \times 1)$ vector containing the random effects that have a normal distribution with a covariance matrix $\omega^{2}$; other distributions for random effects exist [14].

2.1. Estimation of Population Parameters. Since the PopPK data are longitudinal, we can assume that the individuals are sampled randomly from the population, and therefore it is practical to assume that the $\eta_{i}$ 's are also randomly sampled (even though they are not observable) to conduct the estimations of the parameters of (4) and (5) by optimizing the likelihood function defined by the following equation:

$$
L(\theta, \Omega, \Sigma \mid \mathbf{y}) \triangleq p(\mathbf{y} \mid \beta, \Omega, \Sigma)
$$

where $L(\cdot)$ is the likelihood function, $\Omega$ is the variancecovariance matrix of all the $\eta_{i}$ 's, and $\Sigma$ the residual variancecovariance matrix of all the $\varepsilon_{i}$ 's where $p(y \mid \beta, \Sigma, \eta)$ is the conditional probability density of all measurements [15]. If $p(\mathbf{y} \mid \beta, \Sigma, \eta)$ is explicit, the likelihood function $L(\cdot)$ is explicit too, and the exact maximum likelihood estimators can be applied [16], otherwise the estimation of model parameters is made using linear approximations for (6) [17]. Either way, using an explicit likelihood function or an approximation, the precision in the estimated vector $\widehat{\theta}$ depends not only in the residual variability but also in the estimated variance-covariance matrix $\widehat{\Omega}$, which is calculated using the inverse Hessian matrix of the $-\log L(\cdot)$.

\subsection{Nonlinear Mixed-Effects Model Evaluation Criteria.} In the literature referring on the analysis and development of NLME models and PopPK models, the researchers frequently highlighted three statistical criteria to carry out the different evaluations of the model and thus decide which PopPK model they should select within a collection of PopPK models they are considering.

The first two evaluation criteria that we are going to present in this work are the Akaike information criterion (ACI) [18] and the Schwarz's information criterion (SC) [19]. These evaluation criteria are derived from information theory and a Bayesian approach and are commonly used for evaluating nonlinear mixed-effects models when the parameters of the model were estimated by the maximum likelihood method. The AIC criterion provides a balance between the goodness of the fit of the model and the number of parameters required to obtain the fit [20] as is shown in the following equation:

$$
\mathrm{AIC}=N \cdot \log (\mathrm{OFV})+2 \cdot \mathrm{NP},
$$

where $N$ is the number of observations and NP the number of parameters. The final and optimal model is the one with the smallest AIC value. Unlike the AIC criterion, the SC criterion accepts equal probability for each model and for every possible parameter value under the model as is shown in the following equation:

$$
S C=N \cdot \log (\mathrm{OFV})+\mathrm{NP} \cdot \log \mathrm{NP} .
$$

The third evaluation criterion is the estimated error variance also known as the mean square error (MSE). This is based on the simple variance estimator shown in the following equation:

$$
\widehat{\sigma}_{i}^{2}=\frac{\sum_{i=1}^{m} y_{i}-\widehat{y}}{n} .
$$

The use of the MSE is due to the residuals, and it also contains essential information on the quality of the model. Specifically, the estimated variance in the responses, where $y$ is a vector of observed values and $\hat{y}_{i}$, represents a vector of $n$ predictions.

The version of Equation (9) depends on the estimation method used to estimate the PopPK parameters, for example, the variance estimator when the restricted maximum estimation likelihood (REML) is used is shown in the following equation:

$$
\widehat{\sigma}_{i}^{2}=\frac{\sum_{i=1}^{m} y_{i}-\widehat{y}_{\mathrm{REML}_{i}}}{n-p} .
$$

Equation (10) takes into account the degrees of freedom by subtracting the total of parameters $p$ used in the estimation process [14] where $\hat{y}_{\mathrm{REML}_{i}}$ is a vector of predicted values applying REML.

\section{Implementation of the Software Robot}

3.1. The Software Robot. The software robot contained a fuzzy expert system that takes the advantage of the human knowledge and their expertise in a given field to create linguistic descriptors for variables and create fuzzy sets that allow to control the behavior of phenomena under study or even as a tool for decision making [21]. In this work, we developed a software robot as shown in Figure 2 to evaluate indistinct versions of a PopPK model for an aminoglycoside antibiotic named tobramycin which has been the subject of many studies due to its narrow therapeutic window [22]. In this figure, first, we defined the number $N$ of PopPK models to be developed taking into account the number of parameters and covariates. After that, we proceed to establish one by one the $N$ PopPK models. Three statistical evaluation criteria are performed on each model, and they are AIC, SC, and MSE. The software robot uses them as the inputs for the fuzzy expert system, and it collects each defuzzified output into an array until all the $N$ models have been evaluated. Then, the software robot chooses the best PopPK model which is the one that had the lowest value in the fuzzy evaluation, and this will be selected as the best PopPK model.

Thus, the evaluation made by our software robot will guide us to decide if a PopPK model of tobramycin is the best within a group of PopPK models of Tobramycin.

The variables are the evaluation criteria AIC, SC, and MSE presented in Equations (7), (8), and (10), respectively, 


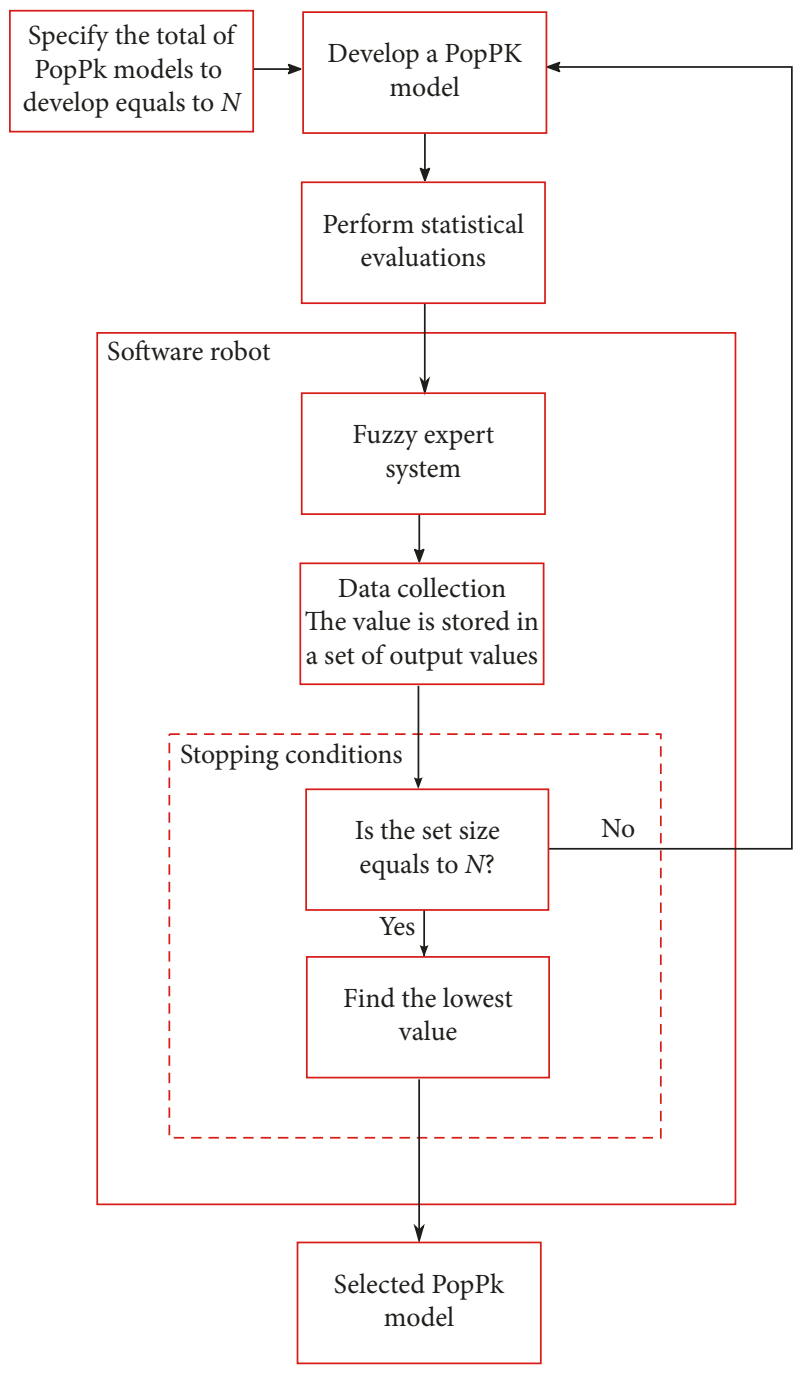

FIgURE 2: Block diagram.

and commonly used to evaluate a PopPK model, and their linguistic descriptors are the ranges of values. For example, the variable AIC has a range of values from 670 to 672.5 considered "Very Low", from 670 to 675 considered "Low," from 672.5 to 677.5 considered "Medium," from 675 to 680 considered "High," and from 677.5 to 680 considered "Very High." "Very Low," "Low," "Medium," "High," and "Very High" are the linguistic descriptors for the sets of values for the variable AIC.

As is typical of the fuzzy theory, the sets of values overlap, and in this way, a value may partially belong to a set and have a degree of membership $0 \leq \mu \leq 1$ that is any place between zero and one, where $\mu$ represents the degree of membership. Thus, the value belongs to several sets with the total membership adding to one. Going back to the above example, the linguistic descriptors of "Very Low" and "Low" are two fuzzy sets for the variable AIC that may overlap, so an AIC of 670.5 could be mostly "Very Low" with $\mu=0.8$ and somewhat "Low" with $\mu=0.2$.

Implementing fuzzy sets with linguistic descriptors to perform a generalization of the output evaluation criteria, results of the fitted PopPK model, we may establish relationships between variables to evaluate the PopPK model by extracting fuzzy rules in terms used by a human expert of the form IF-THEN. In other words, a fuzzy system can perform a description of the phenomena under study, based on the antecedents and consequents presented in a fuzzy rule, that is in the form IF condition THEN action rules [23].

Using the three evaluation criteria AIC, SC, and MSE, we can set a knowledge base in such way that IF AIC is mostly "Very Low" AND CS is mostly "Very Low" AND MSE is also mostly "Very Low" THEN Evaluation is "OPTIMAL". That is, under the fuzzy logic, we are not only going to have linguistic descriptors as the inputs of the fuzzy system but also output variables.

In summary, what a fuzzy system does is to transform crisp input values into fuzzy inputs through a fuzzification unit that establishes the degree of membership to the fuzzy sets for previously defined variables. Then, the fuzzy system uses a rulebase designed by the human expert to predict the fuzzy output of the phenomenon under study, whereby the fuzzy system has a defuzzification unit that transforms the fuzzy output into a crisp value. For this work, we created a fuzzy system with three crisp input variables as shown in Figure 3, the Mamdani max-min inferences to obtain the membership functions of the system and the centroid method to defuzzify the output.

3.2. Fuzzification. As we mentioned, the values of the three evaluation criteria for a developed PopPK model are used to perform a comparison between models, so we can finally decide the PopPK model that is "better," which leads the researchers to deal with an MCDM problem. As an example, a researcher can get to the point in which he needs to decide between two models that have the best evaluation criteria values (Table 1).

In the example of Table 1, the model 2 has a better result in the AIC than model 1 for 1.866 units and in the MSE for 0.048 units. However, model 1 performs better in CS for a significant difference of almost 3 units (2.794). This type of ambiguity in the statistical results and the pressure to get the optimal PopPK model can result in a misinterpretation of the evaluation criteria to select the optimal PopPK model.

In this work, the values of the linguistic variables AIC, CS, and MSE calculated from the development of indistinct versions of a PopPK model of tobramycin were used as our fuzzy input sets. Their maximum and their minimum values are shown in Table 2.

The membership function for the three input variables is tagged as "Very Low," "Low," "Normal," "Medium," "High," and "Very High," and their corresponding parameter values are shown in Table 3.

The membership functions for the output variable "Evaluation" are represented by five membership functions, and the corresponding types and parameter values are shown in Table 4 .

3.3. Fuzzy Rulebase. We created the fuzzy rulebase using the human knowledge of an expert who judged "how much 


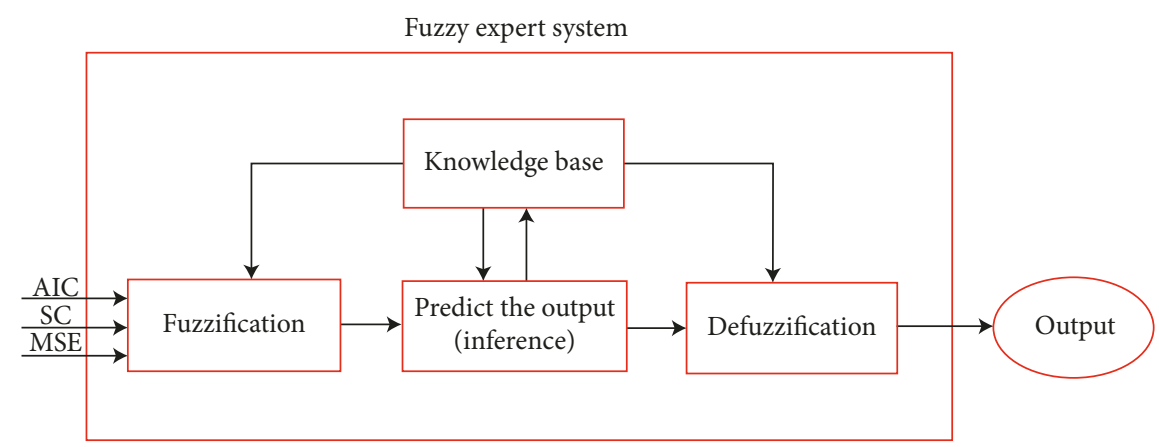

FIgURE 3: The crisp input values AIC, SC, and MSE are transformed into fuzzy inputs by the fuzzification unit which assigns the degree of membership to the fuzzy sets defined for the variables. The fuzzy system can then make inferences or predict outputs of the system by applying knowledge from the expert. Finally, the defuzzification unit transforms the obtained fuzzy output into a crisp value.

TABLE 1: Example of the final evaluation results for a PopPK model.

\begin{tabular}{lccc}
\hline PopPK model & AIC & CS & \\
\hline 1 & 652.470 & 665.300 & MSE \\
2 & 650.604 & 668.094 & 0.14 \\
\hline
\end{tabular}

The best results are the values with red color.

TABLE 2: AIC, CS, and MSE values used for the fuzzy expert system.

\begin{tabular}{lcr}
\hline Variable & Minimum value & Maximum value \\
\hline AIC & 670 & 680 \\
CS & 680 & 795 \\
MSE & 0.095 & 0.14 \\
\hline
\end{tabular}

TABle 3: Parameters of the membership function values for the input variables.

\begin{tabular}{|c|c|c|c|c|c|}
\hline \multirow{2}{*}{ Linguistic variables } & \multicolumn{5}{|c|}{ Linguistic term names, type shapes, and parameters } \\
\hline & Very Low trapezoidal & Low triangular & Medium triangular & High triangular & Very High trapezoidal \\
\hline AIC & {$\left[\begin{array}{llll}670 & 670 & 670.3 & 672.5\end{array}\right]$} & {$\left[\begin{array}{lll}670 & 672.5 & 675\end{array}\right]$} & {$\left[\begin{array}{lll}672.5 & 675 & 677.5\end{array}\right]$} & {$\left[\begin{array}{lll}675 & 677.5 & 680\end{array}\right]$} & {$\left[\begin{array}{lllllll}677.5 & 679.7 & 680 & 680\end{array}\right]$} \\
\hline CS & {$\left[\begin{array}{lllll}676.6 & 679.6 & 680.4 & 683.4\end{array}\right]$} & {$\left[\begin{array}{llll}680 & 683.8 & 687.5\end{array}\right]$} & {$\left[\begin{array}{lll}683.8 & 687.5 & 691.3\end{array}\right]$} & {$\left[\begin{array}{lll}687.5 & 691.3 & 695\end{array}\right]$} & {$\left[\begin{array}{llll}691 & 695 & 695 & 698\end{array}\right]$} \\
\hline MSE & {$\left[\begin{array}{llll}0.092 & 0.092 & 0.09397 & 0.104\end{array}\right]$} & {$\left[\begin{array}{llll}0.0921 & 0.104 & 0.116\end{array}\right]$} & {$\left[\begin{array}{lll}0.104 & 0.116 & 0.128\end{array}\right]$} & {$\left[\begin{array}{lll}0.116 & 0.128 & 0.14\end{array}\right]$} & {$\left[\begin{array}{llll}0.128 & 0.1384 & 0.14 & 0.14\end{array}\right]$} \\
\hline
\end{tabular}

TABLE 4: Parameters of the membership function values for the output variable.

\begin{tabular}{lccccc}
\hline \multirow{2}{*}{ Linguistic variables } & \multicolumn{5}{c}{ Lingustic term names, type shapes, and parameters } \\
& Optimal trapezoidal & High Acceptable & Acceptable triangular & Low Acceptable triangular & Rejected trapezoidal \\
\hline Evaluation & {$\left[\begin{array}{lllll}0 & 0 & 10 & 30\end{array}\right]$} & {$\left[\begin{array}{lllll}10 & 30 & 50\end{array}\right]$} & {$\left[\begin{array}{lllll}30 & 50 & 70\end{array}\right]$} & {$\left[\begin{array}{lllll}50 & 70 & 90\end{array}\right]$} & {$\left[\begin{array}{llll}70 & 90 & 100 & 100\end{array}\right]$} \\
\hline
\end{tabular}

more significant one attribute is than the other." The inference is made on the rules $I F-T H E N$; one rule for each fuzzy output set. The total of rules stems from the heuristic $R=l^{n}$, where $R$ is equal to the total of rules, $l$ is equal to the number of linguistic descriptors, and $n$ represents the number of input variables; thus in our case with $l=5$ and $n=3$ results in a total of 125 rules (Table 5).

3.3.1. Defuzzifier. The fuzzified functions obtained from fuzzy inference are converted into numeric values, for example:

IF the AIC is Low (677)

AND CS is Low (686)

AND MSE is High (.12)
THEN the PopPK model is Low Acceptable (75.2)

The above is achieved by applying the method of center of gravity defuzzifier for $N$ rules using the formula:

$$
C=\frac{\sum_{i=1}^{N} b_{i} \int \mu(i)}{\sum_{i=1}^{N} \int \mu(i)}
$$

where $C$ represents the crisp output value, $b_{i}$ represents the center of the membership function of the consequent of the rule $i$, and $\int \mu(i)$ is the area under the membership function $\int \mu(i)$ of the consequent in the rule $i$. In other words, the center of gravity method calculates the center of mass from the output membership functions.

Figure 4 shows the global behavior of the fuzzy system for the relation between the input variables CS and AIC, 
TABle 5: Fuzzy rules.

\begin{tabular}{|c|c|c|c|c|}
\hline \multirow{2}{*}{ Rule number } & \multicolumn{2}{|c|}{ Linguistic inputs } & \multicolumn{2}{|c|}{ Linguistic output } \\
\hline & AIC & CS & MSE & Evaluation \\
\hline 1 & Very Low & Very Low & Very Low & Optimal \\
\hline 2 & Very Low & Very Low & Low & High Acceptable \\
\hline 3 & Very Low & Very Low & Medium & Acceptable \\
\hline 4 & Very Low & Very Low & High & Low Acceptable \\
\hline 5 & Very Low & Very Low & Very & Optimal \\
\hline 7 & Very Low & Low & Low & High Acceptable \\
\hline 8 & Very Low & Low & Medium & Acceptable \\
\hline 9 & Very Low & Low & High & Low Acceptable \\
\hline 10 & Very Low & Low & Very High & Rejected \\
\hline 11 & Very Low & Medium & Very Low & High Acceptable \\
\hline 12 & Very Low & Medium & Low & High Acceptable \\
\hline 13 & Very Low & Medium & Medium & Low Acceptable \\
\hline 14 & Very Low & Medium & High & Low Acceptable \\
\hline 15 & Very Low & Medium & Very High & Rejected \\
\hline 16 & Very Low & High & Very Low & Acceptable \\
\hline 17 & Very Low & High & Low & Acceptable \\
\hline 18 & Very Low & High & Medium & Low Acceptable \\
\hline 19 & Very Low & High & High & Rejected \\
\hline 20 & Very Low & High & Very High & Rejected \\
\hline 21 & Very Low & Very High & Very Low & Low Acceptable \\
\hline 22 & Very Low & Very High & Low & Low Acceptable \\
\hline 23 & Very Low & Very High & Medium & Rejected \\
\hline 24 & Very Low & Very High & High & Rejected \\
\hline 25 & Very Low & Very High & Very High & Rejected \\
\hline 26 & Low & Very Low & Very Low & Optimal \\
\hline 27 & Low & Very Low & Low & Optimal \\
\hline 28 & Low & Very Low & Medium & High Acceptable \\
\hline 29 & Low & Very Low & High & Low Acceptable \\
\hline 30 & Low & Very Low & Very High & Rejected \\
\hline 31 & Low & Low & Very Low & Optimal \\
\hline 32 & Low & Low & Low & High Acceptable \\
\hline 33 & Low & Low & Medium & Acceptable \\
\hline 34 & Low & Low & High & Low Acceptable \\
\hline 35 & Low & Low & Very High & Rejected \\
\hline 36 & Low & Medium & Very Low & Optimal \\
\hline 37 & Low & Medium & Low & High Acceptable \\
\hline 38 & Low & Medium & Medium & Acceptable \\
\hline 39 & Low & Medium & High & Low Acceptable \\
\hline 40 & Low & Medium & Very High & Rejected \\
\hline 41 & Low & High & Very Low & High Acceptable \\
\hline 42 & Low & High & Low & Acceptable \\
\hline 43 & Low & High & Medium & Low Acceptable \\
\hline 44 & Low & High & High & Rejected \\
\hline 45 & Low & High & Very High & Rejected \\
\hline 46 & Low & Very High & Very Low & Acceptable \\
\hline 47 & Low & Very High & Low & Low Acceptable \\
\hline 48 & Low & Very High & Medium & Rejected \\
\hline 49 & Low & Very High & High & Rejected \\
\hline 50 & Low & Very High & Very High & Rejected \\
\hline 51 & Medium & Very Low & Very Low & Optimal \\
\hline 52 & Medium & Very Low & Low & High Acceptable \\
\hline 53 & Medium & Very Low & Medium & Acceptable \\
\hline 54 & Medium & Very Low & High & Low Acceptable \\
\hline 55 & Medium & Very Low & Very High & Rejected \\
\hline 56 & Medium & Low & Very Low & High Acceptable \\
\hline 57 & Medium & Low & Low & High Acceptable \\
\hline 58 & Medium & Low & Medium & Acceptable \\
\hline 59 & Medium & Low & High & Rejected \\
\hline 60 & Medium & Low & Very High & Rejected \\
\hline
\end{tabular}


TABle 5: Continued.

\begin{tabular}{|c|c|c|c|c|}
\hline \multirow{2}{*}{ Rule number } & \multicolumn{2}{|c|}{ Linguistic inputs } & \multicolumn{2}{|c|}{ Linguistic output } \\
\hline & AIC & $\mathrm{CS}$ & MSE & Evaluation \\
\hline 61 & Medium & Medium & Very Low & Acceptable \\
\hline 62 & Medium & Medium & Low & Acceptable \\
\hline 63 & Medium & Medium & Medium & Low Acceptable \\
\hline 64 & Medium & Medium & High & Rejected \\
\hline 65 & Medium & Medium & Very High & Rejected \\
\hline 66 & Medium & High & Very Low & Acceptable \\
\hline 67 & Medium & High & Low & Low Acceptable \\
\hline 68 & Medium & High & Medium & Rejected \\
\hline 69 & Medium & High & High & Rejected \\
\hline 70 & Medium & High & Very High & Rejected \\
\hline 71 & Medium & Very High & Very Low & Low Acceptable \\
\hline 72 & Medium & Very High & Low & Low Acceptable \\
\hline 73 & Medium & Very High & Medium & Rejected \\
\hline 74 & Medium & Very High & High & Rejected \\
\hline 75 & Medium & Very High & Very High & Rejected \\
\hline 76 & High & Very Low & Very Low & Acceptable \\
\hline 77 & High & Very Low & Low & Acceptable \\
\hline 78 & High & Very Low & Medium & Low Acceptable \\
\hline 79 & High & Very Low & High & Rejected \\
\hline 80 & High & Very High & Very High & Rejected \\
\hline 81 & High & Low & Very Low & Low Acceptable \\
\hline 82 & High & Low & Low & Low Acceptable \\
\hline 83 & High & Low & Medium & Rejected \\
\hline 84 & High & Low & High & Rejected \\
\hline 85 & High & Low & Very High & Rejected \\
\hline 86 & High & Medium & Very Low & Low Acceptable \\
\hline 87 & High & Medium & Low & Low Acceptable \\
\hline 88 & High & Medium & Medium & Rejected \\
\hline 89 & High & Medium & High & Rejected \\
\hline 90 & High & Medium & Very High & Rejected \\
\hline 91 & High & High & Very Low & Low Acceptable \\
\hline 92 & High & High & Low & Rejected \\
\hline 93 & High & High & Medium & Rejected \\
\hline 94 & High & High & High & Rejected \\
\hline 95 & High & High & Very High & Rejected \\
\hline 96 & High & Very High & Very Low & Low Acceptable \\
\hline 97 & High & Very High & Low & Low Acceptable \\
\hline 98 & High & Very High & Medium & Rejected \\
\hline 99 & High & Very High & High & Rejected \\
\hline 100 & High & Very High & Very High & Rejected \\
\hline 101 & Very High & Very Low & Very Low & Acceptable \\
\hline 102 & Very High & Very Low & Low & Low Acceptable \\
\hline 103 & Very High & Very Low & Medium & Rejected \\
\hline 104 & Very High & Very Low & High & Rejected \\
\hline 105 & Very High & Very Low & Very High & Rejected \\
\hline 106 & Very High & Low & Very Low & Low Acceptable \\
\hline 107 & Very High & Low & Low & Low Acceptable \\
\hline 108 & Very High & Low & Medium & Rejected \\
\hline 109 & Very High & Low & High & Rejected \\
\hline 110 & Very High & Low & Very High & Rejected \\
\hline 111 & Very High & Medium & Very Low & Low Acceptable \\
\hline 112 & Very High & Medium & Low & Low Acceptable \\
\hline 113 & Very High & Medium & Medium & Rejected \\
\hline 114 & Very High & Medium & High & Rejected \\
\hline 115 & Very High & Medium & Very High & Rejected \\
\hline 116 & Very High & High & Very Low & Rejected \\
\hline 117 & Very High & High & Low & Rejected \\
\hline 118 & Very High & High & Medium & Rejected \\
\hline 119 & Very High & High & High & Rejected \\
\hline
\end{tabular}


TABle 5: Continued.

\begin{tabular}{lcccc}
\hline \multirow{2}{*}{ Rule number } & \multicolumn{2}{c}{ Linguistic inputs } & \multicolumn{2}{c}{ Linguistic output } \\
& AIC & CS & MSE & Evaluation \\
\hline 120 & Very High & High & Very High & Row \\
121 & Very High & Very High & Low & Rejected \\
122 & Very High & Very High & Medium & Rejected \\
123 & Very High & Very High & High & Rejected \\
124 & Very High & Very High & Very High & Rejected \\
125 & Very High & Very High & & \\
\hline
\end{tabular}

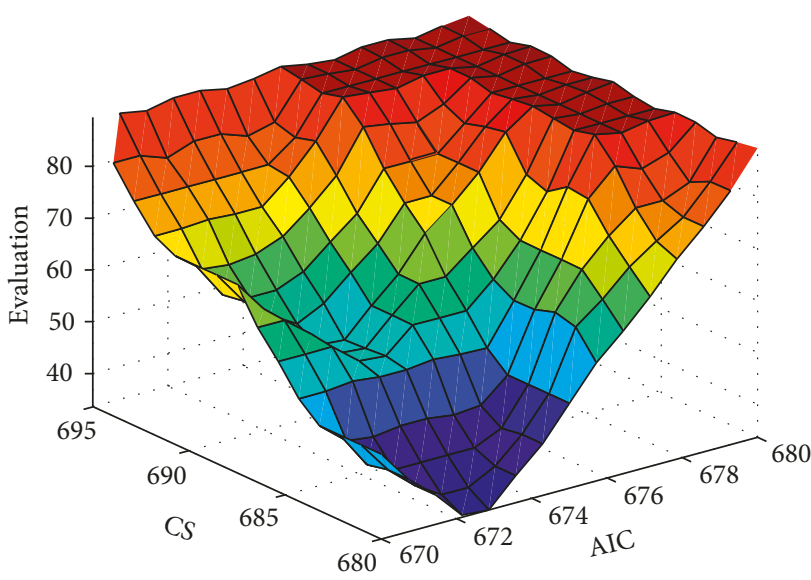

(a)

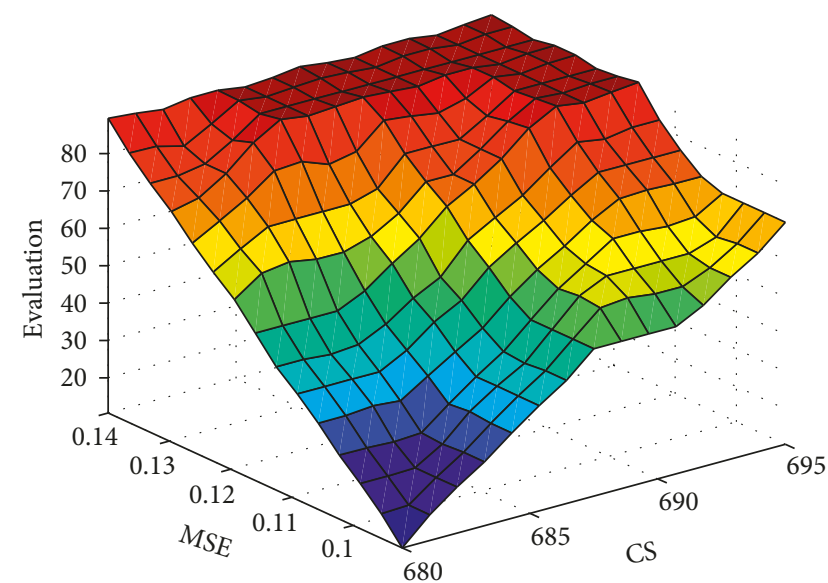

(b)

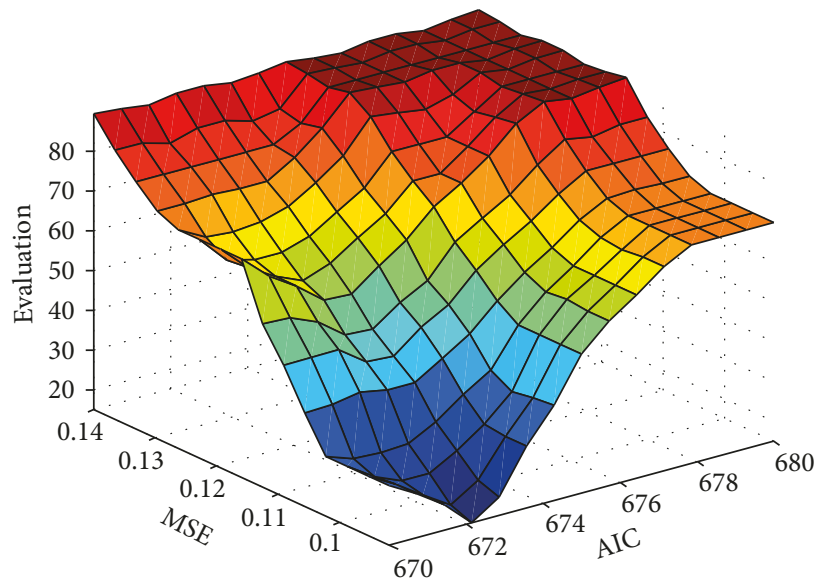

(c)

FIGURE 4: The figure shows the visualization of the system surface when the inputs AIC, CS, and MSE are interacting with each other. (a) The inputs AIC and CS are interacting. (b) The inputs MSE and CS are interacting. (c) The inputs MSE and AIC are interacting.

MSE and CS, and MSE and AIC considering all their rules. For example, if the value of CS is "Very Low," that is 681 and AIC is "High," that is 678.5, then the PopPK model obtains a bad evaluation, or if the value of MSE is "Low," that is 0.1 and the value of CS is also "Low", that is 684, the model obtains an excellent evaluation. Another example will be a scenario where the value of MSE is "Medium," that is 0.12 , and the value of AIC is "Low," that is 674.5 , and the model obtains a good evaluation.

If it was required, the search for the lowest value could be done according to Algorithm 1. The crisp output value can be stored in an array together with other output values taken from other PopPK models that were evaluated previously and then compare them in an automated way to find the lowest value which will determine the optimal PopPK model.

\section{Experimental Results for the Evaluation of Several PopPK Models of Tobramycin}

This section describes the experiments performed to evaluate and compare 21 PopPK models based on the tobramycin database. We developed the PopPK Model of tobramycin in Matlab applying the single compartment model, as it is 


$$
\begin{aligned}
& \text { (1): } i \leftarrow 0 \\
& \text { (2): low } \leftarrow i \\
& \text { (3): for } j=i+1 \text { to total of candidate models }-1 \text { do } \\
& \text { (4): if array } j] \text { is less than array [low] then } \\
& \text { (5): Set low to } j \\
& \text { (6): end if } \\
& \text { (7): end for }
\end{aligned}
$$

Algorithm 1: Find the lowest value algorithm.

shown in Figure 1(a). The covariate model for both $V$ and $\mathrm{Cl}$ were defined as linear.

The estimation of the parameters for all the generated PopPK models was made by applying REML. The optimization process was conducted with the quasi-Newton algorithm using the initial set values for fixed effects of 0.01 for $\mathrm{Cl}$ and 0.01 for $V$ and with a maximum of 100 iterations. The same residual error model was applied to all the experiments. The only variations in the developed PopPK models are the covariate type and the number of parameters included in each model.

4.1. Experiment 1. After having evaluated 21 PopPK models using the evaluations criteria (7), (8), and (10), we end up with the 8 best PopPK models shown in Table 6 .

Once we performed a simple analysis of the 8 PopPK models, we can easily decide that the PopPK models 1 and 2 have the worst values. However, considering the rest of the six PopPK models, it is hard to determine which model is the best given that some of their evaluations values are countered.

4.2. Experiment 2. In this experiment, we used the results of the evaluation criteria shown in Table 6 of the PopPK models $2,4,5,6,7$, and 8 , as input variables in our software robot as described in Section 3. The results of the evaluations made for these PopPK models are shown in Table 7, where now the fuzzy system evaluation (FSE) criteria results are presented for the six selected PopPK models.

\section{Analysis of Results}

The results of experiment 1 of Table 6 represent an example of the type of problem the researcher can face when working in the development of any PopPK model. In this particular case, Table 6 shows that the selection of the best PopPK model is not a trivial decision due to the time consumed to determine which PopPK model obtained the most significant evaluations in comparative with the rest of the models. For example, in Table 6, it can be seen that PopPK model 5 has best AIC evaluation than the PopPK models 4, 6, and 7, but worst evaluation in CS as well as worst evaluation in MSE than the PopPK model 6. The results of experiment 2 of Table 7 show that the fuzzy system gives the worst FSE value

\begin{tabular}{|c|c|c|c|c|c|}
\hline \multirow{2}{*}{$\begin{array}{l}\text { PopPK } \\
\text { Model }\end{array}$} & \multicolumn{5}{|c|}{$\#$ of estimated } \\
\hline & Parameters & Covariates & AIC & CS & MSE \\
\hline 1 & 6 & $(\mathrm{AGE} / V) /(\mathrm{WTCl})$ & 682.04 & 698.2 & 0.0936 \\
\hline 2 & 6 & $(\mathrm{SEX}, \mathrm{AGE} / V)$ & 679.9 & 695 & 0.093 \\
\hline 3 & 7 & $\begin{array}{c}(\mathrm{WT}, \mathrm{AGE} / V) / \\
(\mathrm{WT} / \mathrm{Cl})\end{array}$ & 683.6 & 701.1 & 0.093 \\
\hline 4 & 6 & $(\mathrm{SEX}, \mathrm{AGE} / V)$ & 673.1 & 689.2 & 0.09336 \\
\hline 5 & 9 & $\begin{array}{c}(\mathrm{SEX}, \mathrm{AGE} / V) / \\
(\mathrm{SEX}, \mathrm{AGE}, \mathrm{WT} / \mathrm{Cl})\end{array}$ & 670.4 & 693 & 0.0936 \\
\hline 6 & 7 & $\begin{array}{c}(\mathrm{SEX}, \mathrm{AGE} / V) \\
(\mathrm{SEX} / \mathrm{Cl})\end{array}$ & 672.7 & 691 & 0.093 \\
\hline 7 & 7 & $\begin{array}{l}\text { (WT, AGE, } \\
\text { SEX/Cl) }\end{array}$ & 675.3 & 691.3 & 0.139 \\
\hline 8 & 10 & $\begin{array}{l}(\mathrm{WT}, \mathrm{HT} / V) /(\mathrm{WT}, \\
\mathrm{AGE}, \mathrm{HT}, \mathrm{BMI} / \mathrm{Cl})\end{array}$ & 682.7 & 707.5 & 0.0928 \\
\hline
\end{tabular}
to PopPK model 7 (88.9). The PopPK model 7 has the worst evaluation of AIC, CS, and MSE than the PopPK model 4, which is the model with the best FSE value (31.6). It can be

\begin{tabular}{|c|c|c|c|c|c|c|}
\hline \multirow{2}{*}{$\begin{array}{l}\text { PopPK } \\
\text { Model } \\
\end{array}$} & \multicolumn{6}{|c|}{ \# of estimated } \\
\hline & Parameters & Covariates & AIC & CS & MSE & FSE \\
\hline 2 & 6 & $(\mathrm{WT}, \mathrm{AGE} / V)$ & 679.9 & 695 & 0.093 & 88.4 \\
\hline 4 & 6 & $\begin{array}{c}\text { (SEX,AGE/V) } \\
\quad(\mathrm{SEX},\end{array}$ & 673.1 & 689.2 & 0.09336 & 31.6 \\
\hline 5 & 9 & $\begin{array}{c}\mathrm{AGE} / V) /(\mathrm{SEX}, \\
\mathrm{AGE}, \mathrm{WT} / \mathrm{Cl})\end{array}$ & 670.4 & 693 & 0.0936 & 55.6 \\
\hline 6 & 7 & $\begin{array}{c}(\mathrm{SEX}, \mathrm{AGE} / V) \\
(\mathrm{SEX} / \mathrm{Cl})\end{array}$ & 672.7 & 691 & 0.093 & 34.4 \\
\hline 7 & 7 & $\begin{array}{l}\text { (WT, AGE, } \\
\text { SEX/Cl) }\end{array}$ & 675.3 & 691.3 & 0.139 & 88.9 \\
\hline 8 & 10 & $\begin{array}{c}(\mathrm{WT}, \\
\mathrm{HT} / V) /(\mathrm{WT}, \\
\mathrm{AGE}, \mathrm{HT}, \\
\mathrm{BMI} / \mathrm{Cl})\end{array}$ & 682.7 & 707.5 & 0.0928 & 88.7 \\
\hline
\end{tabular}

TABle 6: Results of the 8 best PopPK model of tobramicyn. The covariates used are weight (WT), age, height (HT), and body mass index (BMI).

Table 7: Summary of all evaluation criteria applied. Model 4 is the best evaluated by the fuzzy system.

seen that the fuzzy system considered the trade-offs among the rest of standard statistical evaluation criteria: AIC, CS, and MSE provided a clearer picture of the order of the worst PopPK model to the best PopPK model.

\section{Conclusions}

Our results bolster the hypothesis that the software robot can be successfully implemented to evaluate PopPK models ensuring the selection of the best PopPK model when the choice of this becomes an MCDC problem. Given that the FSE criteria is built taking into account the classical evaluation criteria (AIC, CS, and MSE), we are able to only use the FSE as our unique automated evaluation criteria. This reduced the time and the error in the selection of the best PopPK model.

Another advantage is that if we want to use the same fuzzy system methodology for the evaluation of a different case study, for example, a PopPK model that involves another type of drug as phenobarbital, or the amount of individuals in the study is different, we only need to evaluate 
a certain amount of models to perform adjustments in the membership function ranges.

Our software robot performs fuzzy evaluations offering a stronger alternative to increase the efficiency in the selection of the best PopPK from a set. It can help the pharmaceutical scientist or the expert researchers in the area of computer intelligence to incorporate, expand, and improve the implementation of this software either in new versions of commercial software or in the development of new software to incorporate human expertise.

The proposed software robot can become a strong support to further studies regarding this novel approach by helping the pharmaceutical scientist or the expert researchers in the area of computer intelligence to incorporate, expand, and improve this development.

\section{Data Availability}

The data of our document are available upon request to any of the authors.

\section{Conflicts of Interest}

The authors declare that they have no conflicts of interest.

\section{Acknowledgments}

The research leading to these results has received funding from Instituto Politécnico Nacional (IPN), under the Research Project SIP20180499, as well as the Commission of Operation and Promotion of Academic Activities of IPN (COFAA), and the National Council of Science and Technology (CONACYT).

\section{References}

[1] S. Rosenbaum, Basic Pharmacokinetics and Pharmacodynamics: An Integrated Textbook and Computer Simulations, Wiley, Hoboken, NJ, USA, 2012.

[2] G. Fitzmaurice, M. Davidian, V. Geert, and G. Molenberghs, Longitudinal Data Analysis, Chapman and Hall/CRC., Boca Raton, FL, USA, 1st edition, 2008.

[3] J. Gabrielsoon and D. Weiner, Pharmacokinetic and Pharmacodynamic Data Analysis, Concepts and Applications, Swedish Pharmaceutical Press, Sweden, Europe, 4th edition, 2007.

[4] K. Vučićević, B. Miljkovic, S. Vezmar Kovačević, Z. Todorovic, M. Prostran, and I. Grabnar, Population Pharmacokinetic Analysis of Therapeutic Drug monitoring Data Otimizing Pharmacotherapy of Antiepileptic Drugs, Intech, London, UK, 1st edition, 2011.

[5] F. Mentré and S. Escolano, "Prediction discrepancies for the evaluation of nonlinear mixed-effects models," Journal of Pharmacokinetics and Pharmacodynamics, vol. 33, no. 3, pp. 345-367, 2006.

[6] P. J. Williams and E. I. Ette, "Determination of model appropriateness," in Simulation for Designing Clinical Trials: A Pharmacokinetic-Pharmacodynamic Modeling Prospective, H. C. Kimko and S. B. Duffull, Eds., Marcel Dekker, New York, NY, USA, pp. 68-96, 2003.

[7] A. Farkas, G. Daroczi, P. Villasurda, M. Dolton, M. Nakagaki, and J. A. Roberts, "Comparative evaluation of the predictive performances of three different structural population pharmacokinetic models to predict future voriconazole concentrations," Antimicrobial Agents and Chemotherapy, vol. 60, no. 11, pp. 6806-6812, 2016.

[8] K. Brendel, E. Comets, C. Laffont, C. Laveille, and F. Mentré, "Metrics for external model evaluation with an application to the population pharmacokinetics of gliclazide," Pharmaceutical Research, vol. 23, no. 9, pp. 2036-2049, 2006.

[9] A. Lazim, "Fuzzy multi criteria decision making and its applications: a brief review of category," Procedia-Social and Behavioral Sciences, vol. 97, pp. 131-136, 2013.

[10] E. P. Klement and W. Slany, "Fuzzy logic in artificial intelligence," in Proceedings of IJCAI'97 Workshop Nagoya, pp. 179-190, Nagoya, Japan, August 1997.

[11] R. Kunhimangalam, S. Ovallath, and P. Joseph, "A novel fuzzy expert system for the identification of severity of carpal tunnel syndrome," BioMed Research International, vol. 2013, Article ID 846780, 12 pages, 2013.

[12] F. Gaxiola, P. Melin, F. Valdez, and O. Castillo, "Generalized type-2 fuzzy weight adjustment for backpropagation neural networks in time series prediction," Information Sciences, vol. 325, pp. 159-174, 2015.

[13] G. C. Pillai, F. Mentré, and J.-L. Steimer, "Non-linear mixed effects modeling - from methodology and software development to driving implementation in drug development science," Journal of Pharmacokinetics and Pharmacodynamics, vol. 32, no. 2, pp. 161-183, 2005.

[14] M. Davidian and D. M. Giltinam, Nonlinear Models for Repeated Measurement Data, Chapman and Hall, Boca Raton, FL, USA, 1995.

[15] M. Lavielle, Mixed Effects Models for the Population Approach Models, Tasks, Methods and Tools, Chapman and Hall/CRC Press, Boca Raton, FL, USA, 2014.

[16] S. Donnet and A. Samson, "A review on estimation of stochastic differential equations for pharmacokineticl pharmacodynamic models," Advanced Drug Delivery Reviews, vol. 65, no. 7, pp. 929-939, 2013.

[17] Y. Wang, "Derivation of various nonmem estimation methods," Journal of Pharmacokinetics and Pharmacodynamics, vol. 34, no. 5, pp. 575-593, 2007.

[18] H. Akaike, "A bayesian analysis of the minimum aic procedure," Annals of the Institute of Statistical Mathematics, vol. 30, no. 1, pp. 9-14, 1978.

[19] G. Schwarz, "Estimating the dimension of a model," The Annals of Statistics, vol. 6, no. 2, pp. 461-464, 1978.

[20] R. Gieschke and D. Serafin, Development of Innovative Drugs via Modeling with MATLAB, Springer, Berlin, Heidelberg, Germany, 2014.

[21] Z. Gong, J. Forrest, and T. Yao, Uncertain Fuzzy Preference Relations and Their Applications, Vol 281, Springer-erlag, Berlin, Heidelberg, Germany, 2013.

[22] D. Xuan, J. F. Lu, D. P. Nicolau, and C. H. Nightingale, "Population pharmacokinetics of tobramycin in hospitalized patients receiving once-daily dosing regimen," International Journal of Antimicrobial Agents, vol. 15, no. 3, pp. 185-191, 2000.

[23] J. Espinosa, J. Vandewalle, and V. Wertz, Fuzzy Logic, Identification and Predictive Control (Advances in Industrial Control), Springer-Verlag, Secaucus, NJ, USA, 1st edition, 2004. 


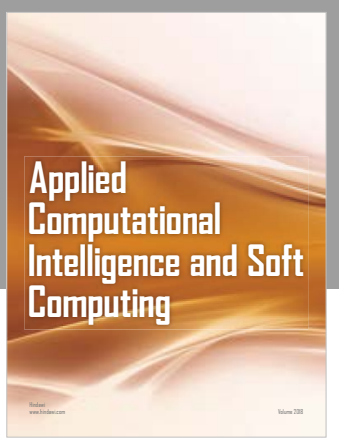

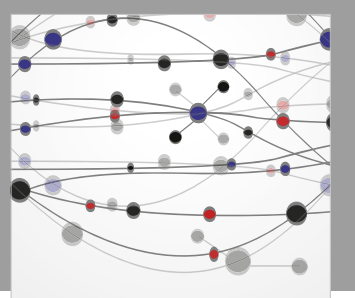

The Scientific World Journal
Submit your manuscripts at

Computing
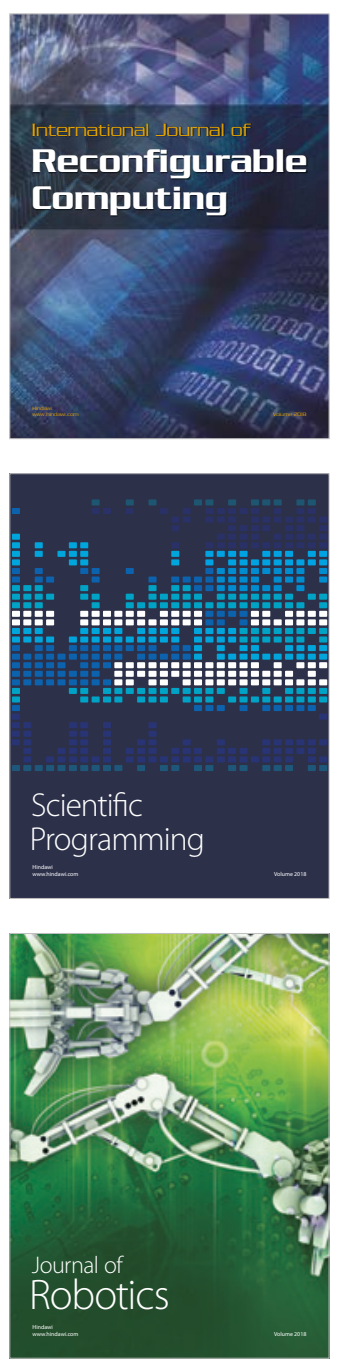

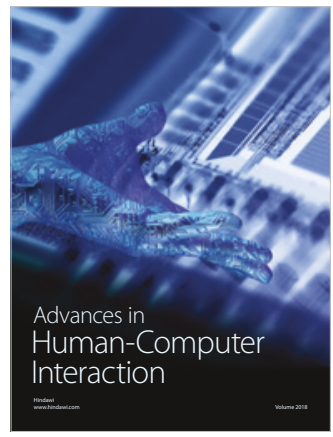

Human-Compute

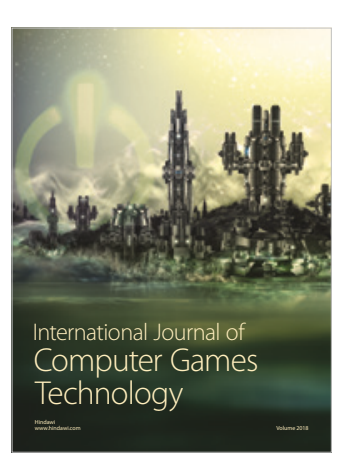

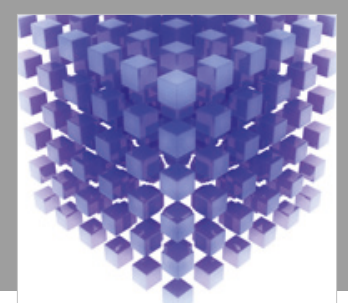

Mathematical Problems in Engineering

\section{Engincering}
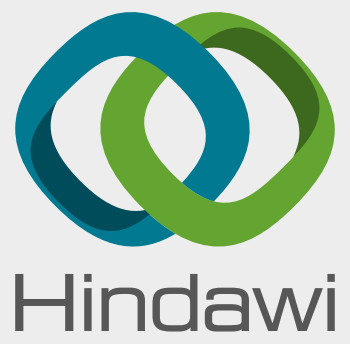

www.hindawi.com
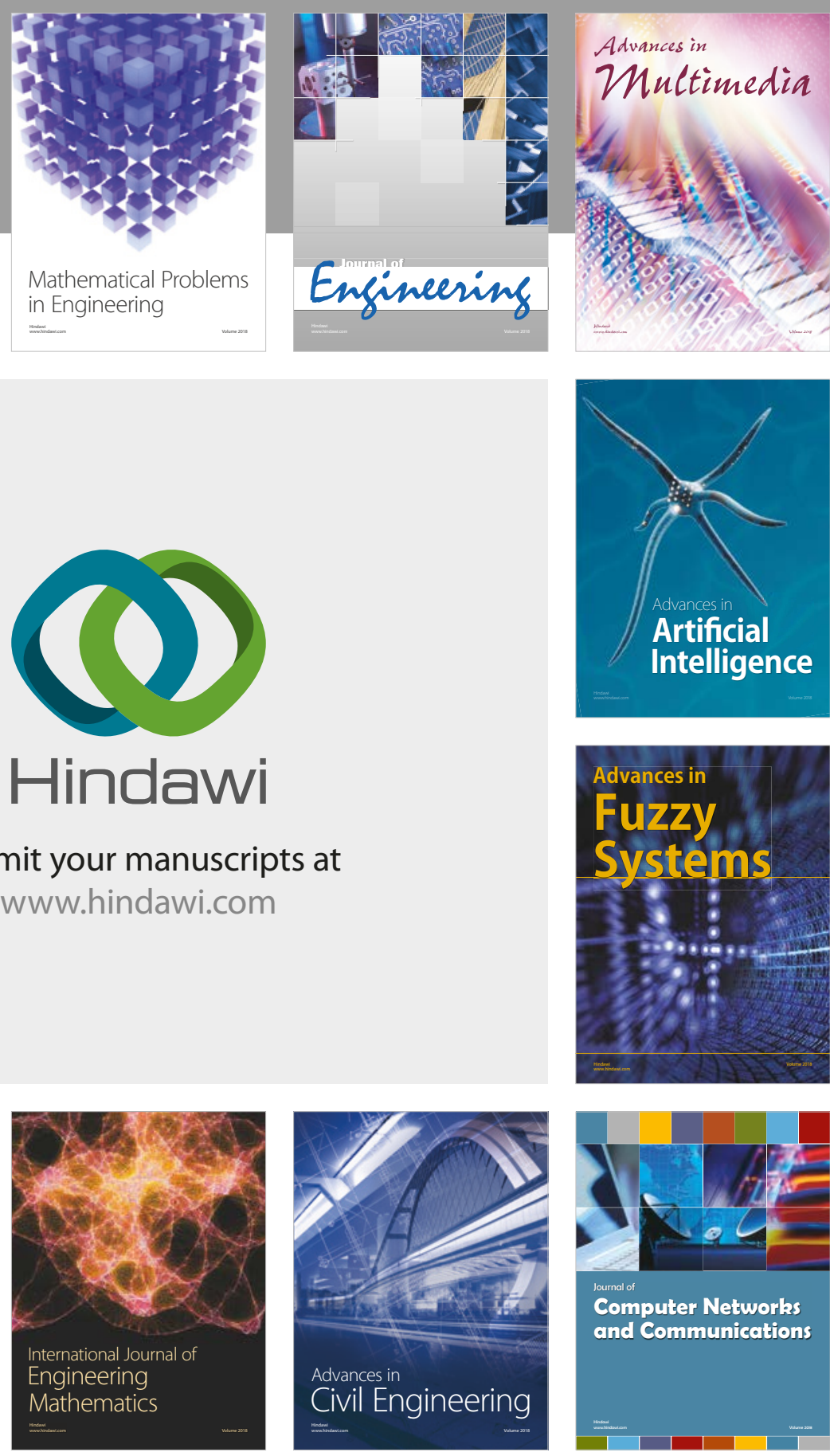

Computer Networks and Communications

Multimedia
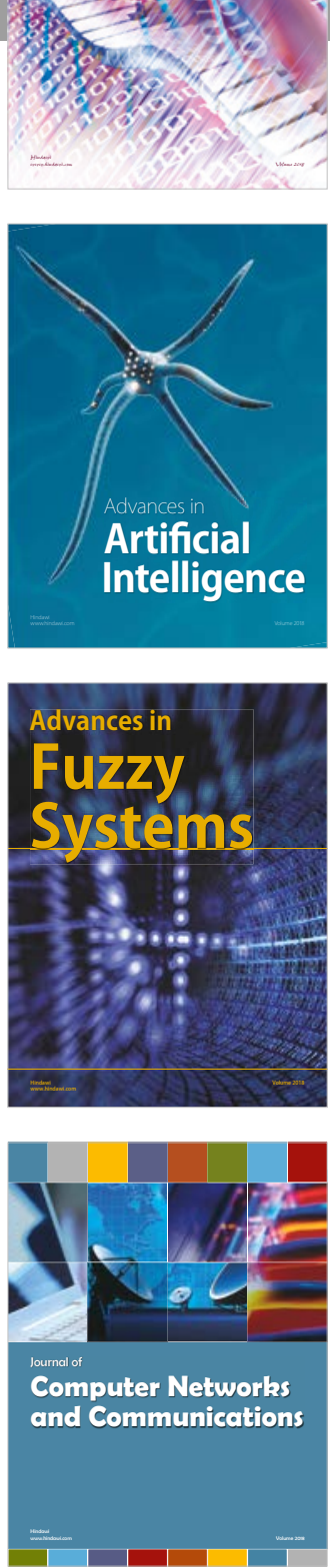

Advances in

Modelling \&

Simulation

in Engineering

interaction

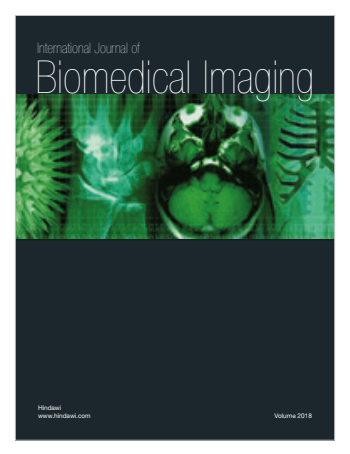

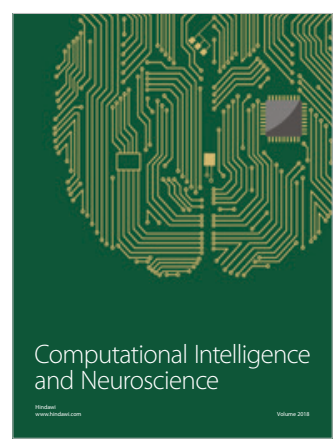

